\title{
Editorial
}

Published online: September 16, 2015

\section{History and Development of Tsuchiya General Hospital in Hiroshima}

\section{Hideki Kawanishi}

Department of Artificial Organs and Surgery, Tsuchiya General Hospital, and Faculty of Medicine, Hiroshima University, Hiroshima, Japan

\section{About Hiroshima and Tsuchiya General Hospital}

With a population of just over 1 million, Hiroshima is one of the major cities in Western Japan. On August 6, 1945, an atomic bomb was detonated approximately 600 meters above the center of the city, resulting in a blast that instantaneously reduced a thriving city from a population of 420,000 people to barren scorched earth. Ironically, the places related to this tragedy are now the main tourist attractions, and people from all over the world visit Peace Memorial Park and the Museum.

Tsuchiya General Hospital is located in front of the Hiroshima Peace Memorial Museum (fig. 1).
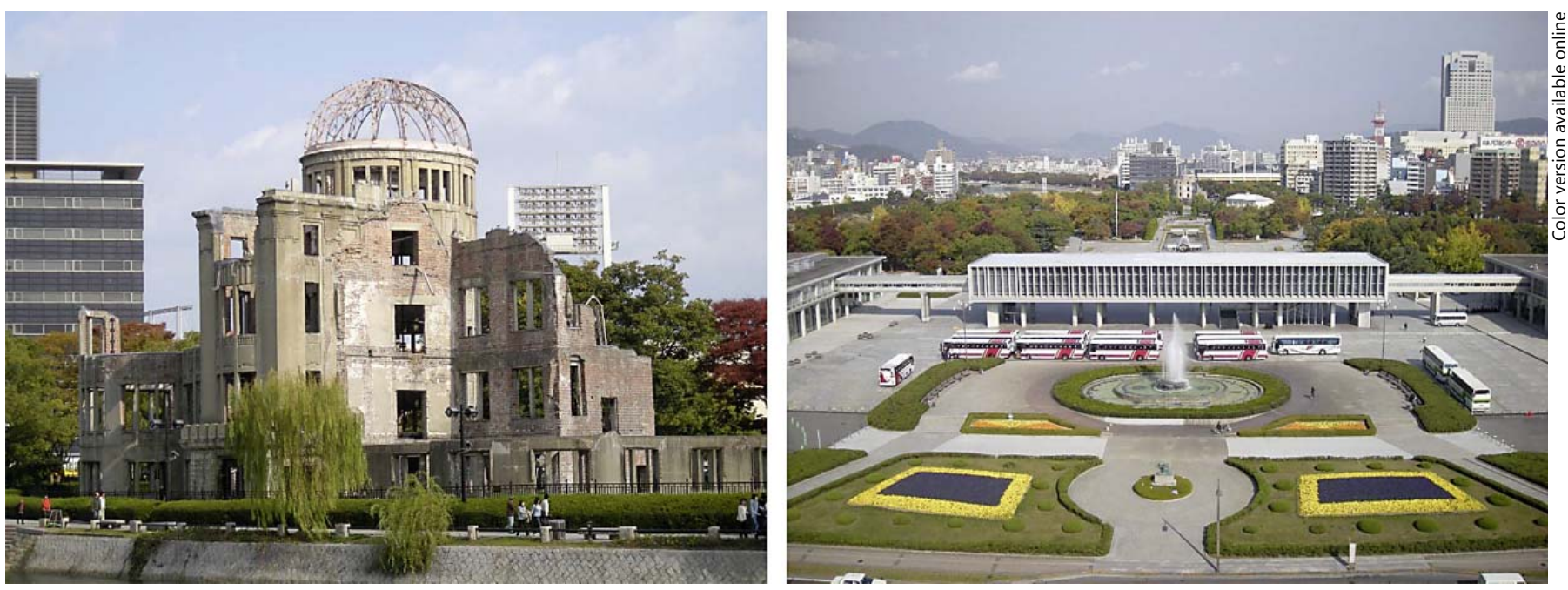

Fig. 1. Atomic bomb dome (left) and peace memorial park (right) in Hiroshima, photos from the hospital roof.

Hideki Kawanishi, MD

Tsuchiya General Hospital

3-30 Nakajima-cho

Naka-ku, Hiroshima 730-8655 (Japan)

E-Mail h-kawanishi@ tsuchiya-hp.jp 

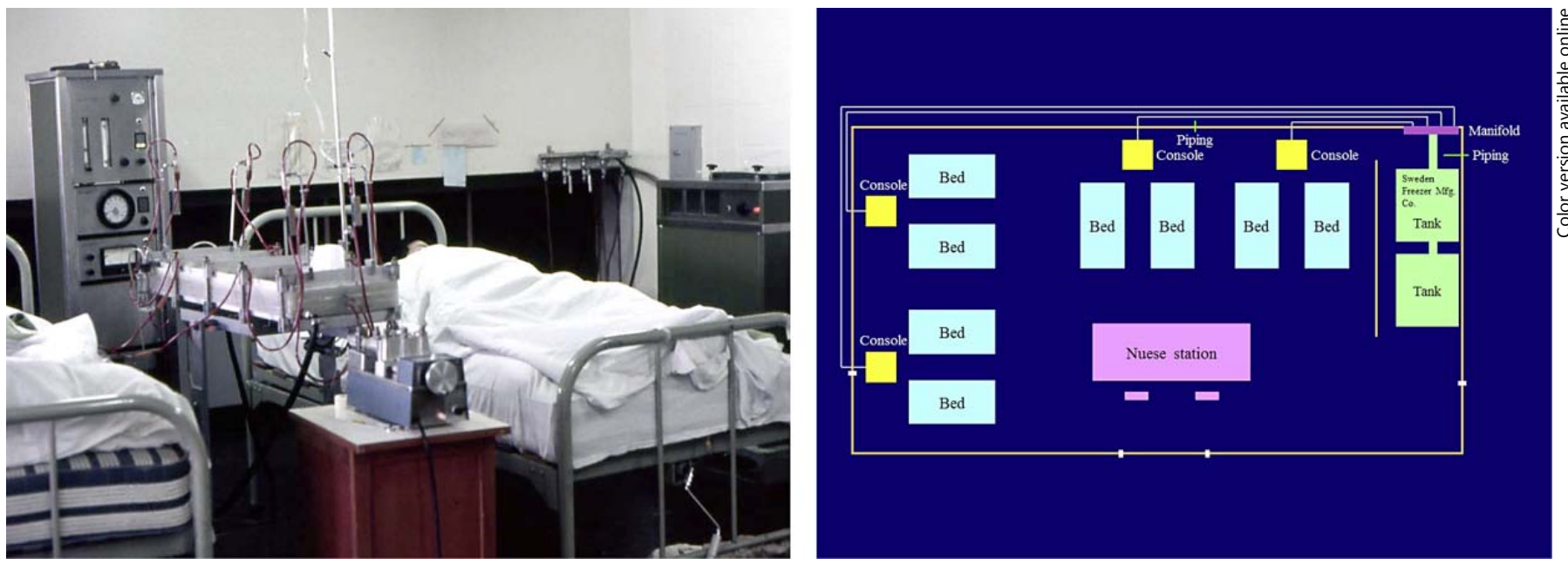

Fig. 2. Sweden freezer-bedside console, and Kiil dialyzer (left), CDDS (right) at 1966.

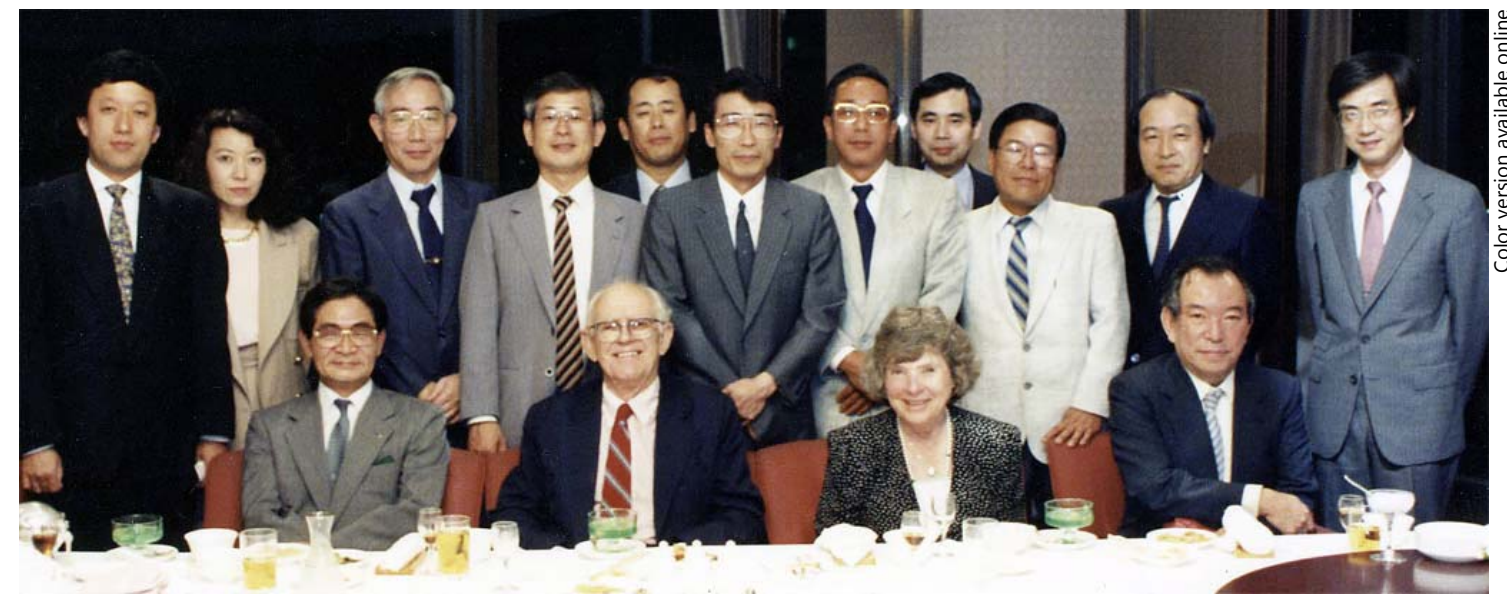

Fig. 3. Dr. Scribner in Hiroshima, 1990, time of ISN Tokyo, Center: Dr. and Mrs. Scribner, right: Dr. Taro Tsuchiya, right edge: Dr. Hideki Kawanishi.

\section{History of Tsuchiya General Hospital}

Tsuchiya hospital was developed as a surgical hospital in 1937. Dr. Taro Tsuchiya, who had worked with the previous president, established the first blood bank in Hiroshima and was a friend of Dr. Belding Scribner, who was a pioneer ofdialysis therapy. Dr. Kiyohiko Dohi(Emeritus, Hiroshima University) was then a young surgeon learning dialysis at the University of Washington in Seattle, 1965.

Subsequently, we opened a hemodialysis (HD) center, making use of the Sweden Seattle-central dialysis fluid delivery system (CDDS), Sweden Freezer-bedside console, and Kiil dialyzer developed by Dr. Scribner (fig. 2, 3). This was the first CDDS in Japan, and HD was performed $24 \mathrm{~h}$ a day, 6 days a week. Thereafter, the number of dialysis patients increased, and as of the end of 2014, 1,300 patients (HD 1,150, peritoneal dialysis (PD) 150) are managed across 1 hospital and 2 clinics. Moreover, Dr. Taro Tsuchiya established JMS Co., Ltd., in 1965, a manufacturer and marketer of disposable medical devices and dialysis systems.

\section{Our Research Deals with Dialysis and Blood Purification}

Dr. Hideki Kawanishi, who received his training as a surgeon, started to develop artificial liver support systems based on adsorbents in the late 1970s. This was the first 
Fig. 4. A new sorbent, urethane sheets embedded with powdered charcoal, developed by Dr. Hideki Kawanishi, 1983.

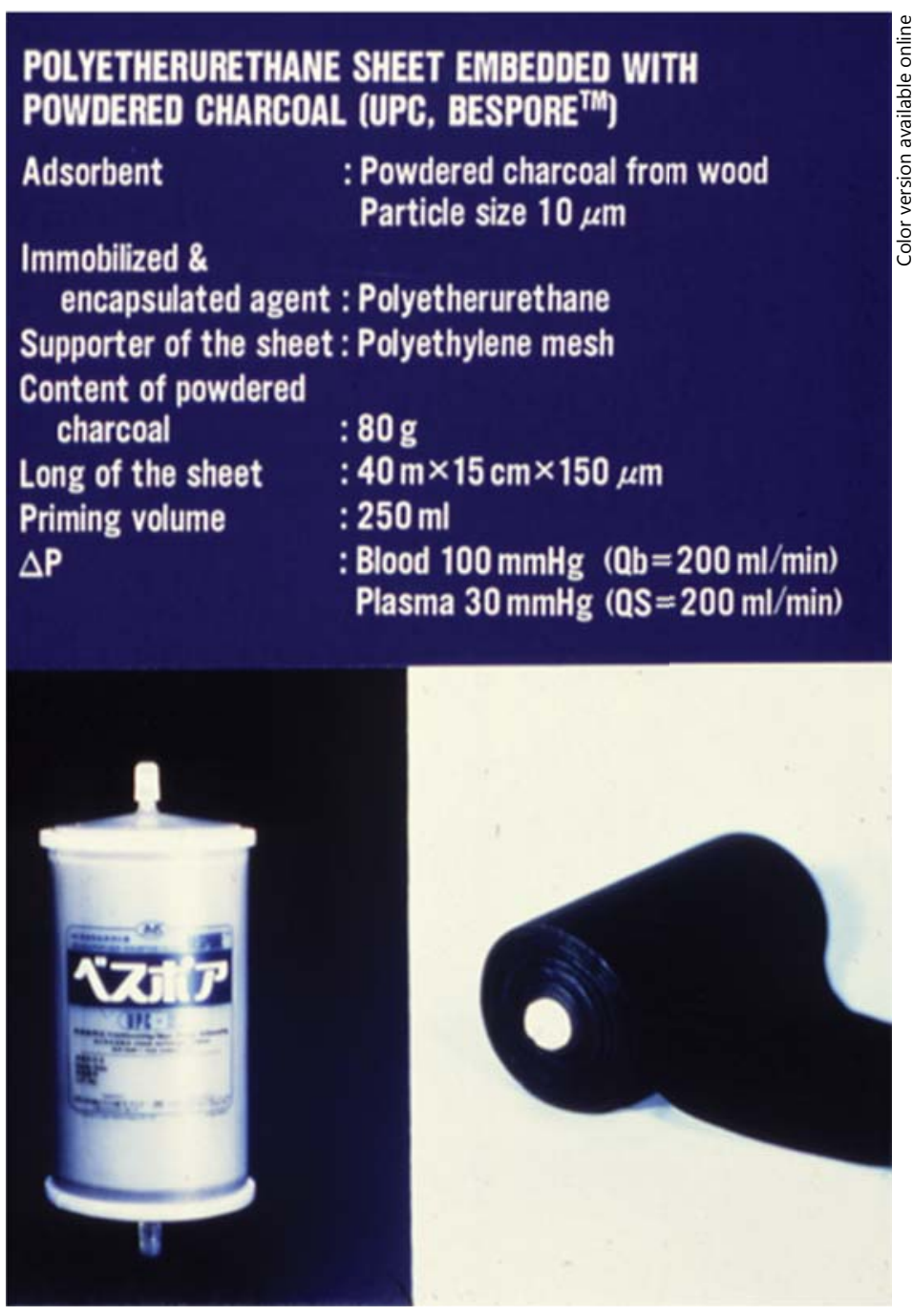

Fig. 5. The Japanese Society for HDF, 1980, left to right: Dr. N.K. Man, Kazuo Ota, Hideki Kawanishi, Yutaka Koda.

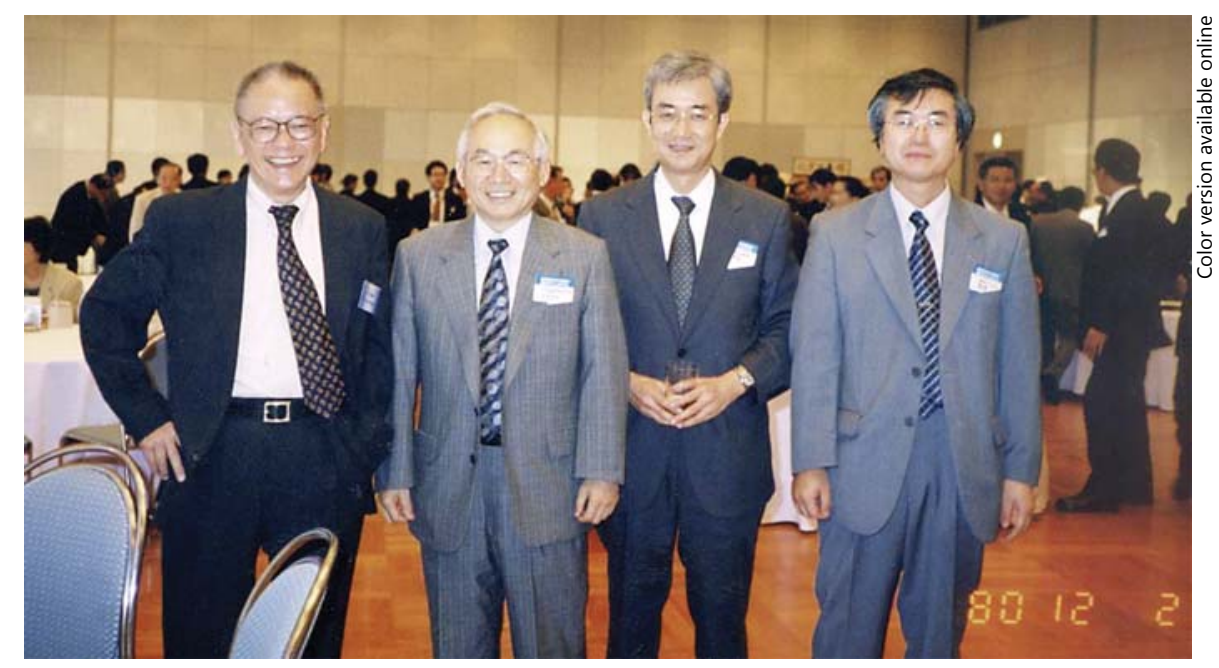




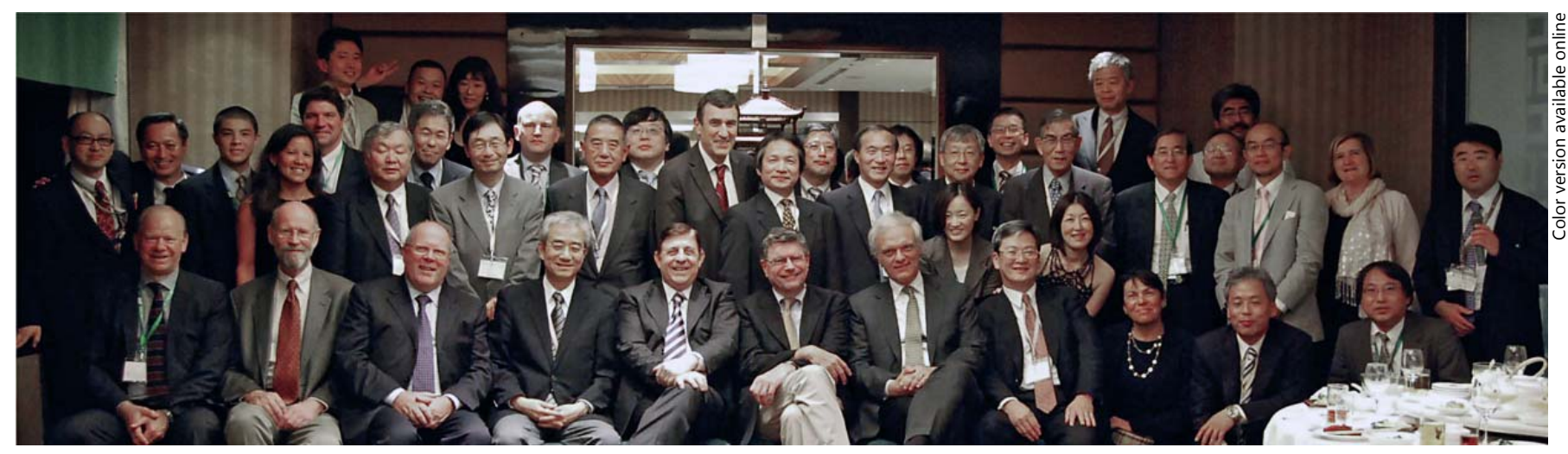

Fig. 6. The international symposium of HDF in the congress of the Japanese society for dialysis therapy 2010, left to right: R. Greewood, R.A. Ward, C. Ronco, H. Kawanishi, F. Locatelli, B. Canaud, R. Vanholder, P.J. Blankestijn with the Japanese team.

stage of his blood purification therapy. A new sorbent consisting of urethane sheets embedded with powdered charcoal worked by adsorbing medium-to-large molecules and protein-bound substances (fig. 4). This was developed for use in liver support systems, and was clinically applied from 1984 to the late 1990s.

Since the introduction of the continuous ambulatory PD system in 1983, which replaced the Baxter system, we have developed the original peritoneal dialysate (Perisate ${ }^{\mathrm{TM}}$ ) and a new connection system (Safedap ${ }^{\mathrm{TM}}$ ) based on research from JMS and also joint research performed in 1987. Later, the introduction of neutral dialysates (low glucose degradation product dialysates) led to the development of various other automatic and semiautomatic back-exchange systems.

The fully automated HD system was developed in 2001 with the aim of standardizing and promoting labor-saving in dialysis treatment. This system uses dialysis fluid back-filtration to perform priming, blood rinse back, and rapid fluid replenishment, and guides the blood into the dialyzer using a drainage pump for ultrafiltration. CDDS combines the processes of creating and supplying dialysis water and dialysis fluid to achieve a level of purity equivalent to that of ultrapure dialysis fluids. This system can be used with online hemodiafiltration (HDF).

Encapsulating peritoneal sclerosis (EPS) is a serious complication of long-term PD. The mortality rate of EPS is high, mainly because of complications related to bowel obstruction. Dr. Hideki Kawanishi developed a surgical technique of total intestinal enterolysis without enterectomy in 1993, and since then we have treated patients believing that surgical therapy is the only curative treatment for EPS. Between 1993 and the end of 2014, we performed

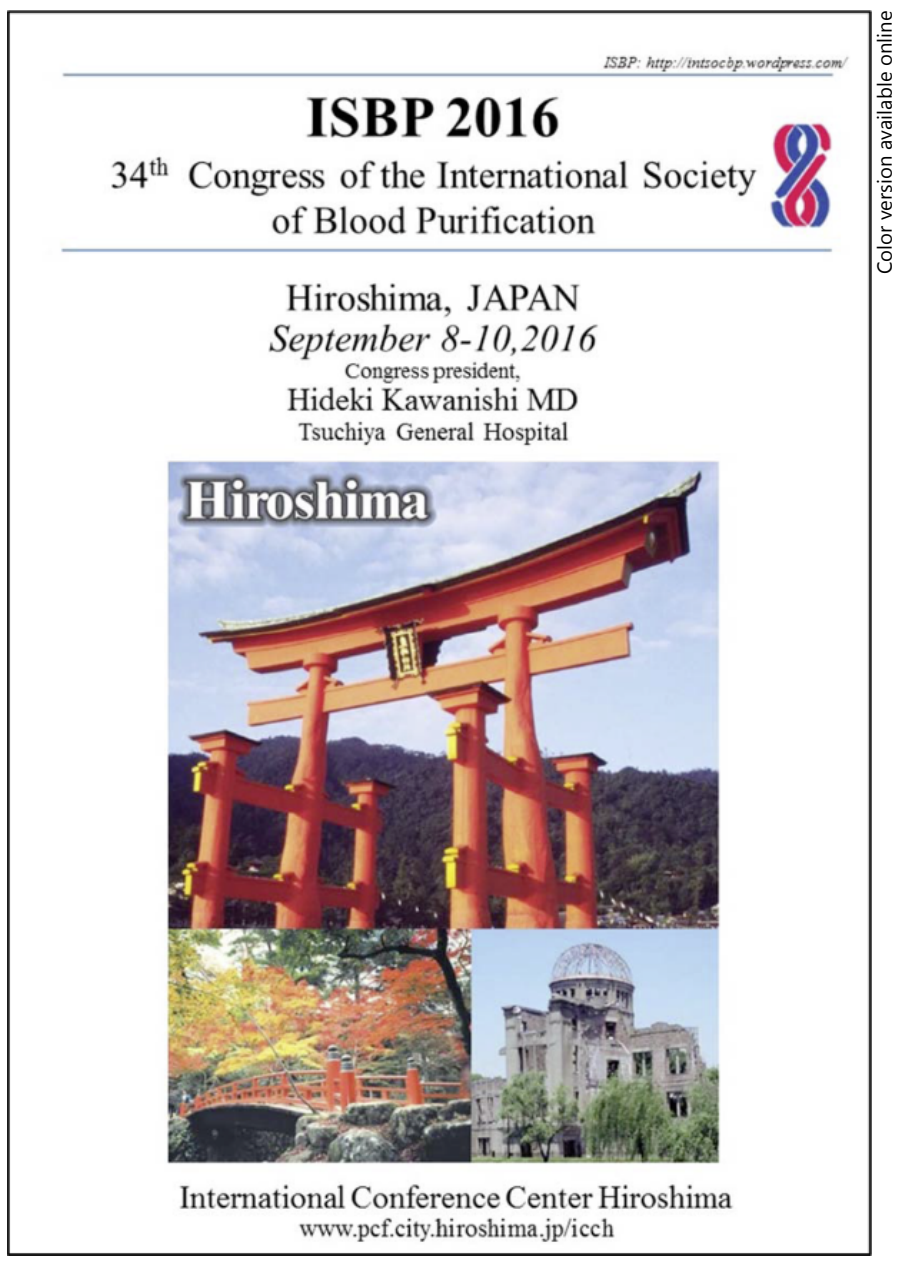

Fig. 7. Thirty fourth ISBP, 2016, in Hiroshima. 
289 enterolysis procedures in 218 patients. These activities have promoted a better understanding of and countermeasures against EPS, and EPS is no longer considered a fatal complication.

\section{Our Active Promotion in the Dialysis Field}

Our team has performed a great deal of research in the dialysis field. The Japanese Society for HDF was established in 1995, and served to disseminate information about online HDF in Japan (fig. 5). The research results were presented at the international symposium of HDF in the congress of the Japanese Society for Dialysis Therapy 2010 (fig. 6). Moreover, reimbursement for medical services in Japan was extended to include online HDF from 2012. The number of patients undergoing HDF is rapidly increasing and currently is $15 \%$ of the total dialysis population in Japan.

In the field of PD, the third Asia chapter meeting of ISPD was held in 2007. The Japanese Society for Dialysis Access was established in 1998, and has been working toward the development of not only vascular access but also peritoneal access technologies. The Japanese Society for Dialysis Access has maintained a cooperative relationship with the current Vascular Access Society and the Vascular Access Society of America. Line feed the International Society of Blood Purification congress is planned to be held at the International Conference Center Hiroshima in September 2016 as the culmination of these activities (fig. 7). We are looking forward to seeing you in Hiroshima, and hope you will enjoy the meeting! 